\title{
Implementation of Accurate Broadband Steering Vectors for Broadband Angle of Arrival Estimation
}

\author{
MA Alrmah*, S Weiss*, JG McWhirter \\ ${ }^{*}$ Department of Electronic and Electrical Engineering, University of Strathclyde, Glasgow, Scotland \\ ${ }^{\dagger}$ School of Engineering, University of Cardiff, Cardiff, Wales, UK \\ \{mohamed.alrmah,stephan.weiss\}@strath.ac.uk, mcwhirterjg@cardiff.ac.uk
}

\begin{abstract}
Motivated by accurate broadband steering vector requirements for applications such as broadband angle of arrival estimation, we review fractional delay filter designs. A common feature across these are their rapidly decreasing performance as the Nyquist rate is approached. We propose a filter bank based approach, which operates standard fractional delay filters on a series of frequency-shifted subband signals, such that they appear in the filters' lowpass region. We demonstrate the appeal of this approach in simulations.
\end{abstract}

\section{Introduction}

For broadband array signal processing, the time delays arising from signal wave fronts travelling across the array at finite speed cannot be represented by phase shifts as in the narrowband case but require to be addressed as lags. Since these delays are normally not integer multiples of the sampling interval, fractional delay filters need to be used $[1,2]$. With broadband sensor array applications potentially operating across several octaves, the implementation accuracy of such fractional delay is crucial to the accuracy of broadband angle of arrival estimation or the performance of any other subsequent processing [3].

Fractional delays can be implement by appropriately a sampled sinc function $[4,5]$. To achieve a finite filter, truncation is employed to create a discrete prolate spheroidal sequence [6], which compared to an ideal delay system cause ripple in the group delay and an increasingly poor approximation with increasing frequency. This leads to a restricted accuracy of the fractional delay filter [5], and a limitation of its application to lowpass-type signals.

To improve the performance of fractional delay filters, tapered windows instead of rectangular ones have been proposed for the truncation of the sinc [4,7], which leads to a reduced group delay ripple. An entirely different approach is based on polynomial approximation was proposed by Farrow [8], which provides relatively good accuracy at a modest filter order. However, both windowed sinc and Farrow structure still perform best at low frequencies, and degrade significantly in higher frequency ranges.

Filter banks have been used in the context of fractional delays previously, since subband processing can shorten the long impulse responses found when sampling a sinc off the zerocrossings [9]. By recognising that most current fractional delay approaches are reasonably accurate in the low frequency range and only break down at higher frequencies, in this paper we proposed to use the filter bank approach to modulate undecimated subbands to adopt lowpass characteristics. After applying accurate fractional delay filters in the lowpass domain, a frequency shift to the original band and a synthesis filter bank operation complete our proposed accurate broadband fractional delay filter approach.

The paper is organised as follows. Sec. 2. motivates the requirement of highly accurate fractional delay filters by reviewing the construction of broadband steering vectors. Sec. 3. reviews different approaches for designing fractional delay filters. Thereafter, our proposed filter bank approach is outlined in Sec. 4.. The complexity of various fractional delay filter implementation methods is then analysed and compared in Sec. 5.. Simulation results are provided in Sec. 6. to demonstrate and compare the accuracy of our proposed approach to various benchmarks. Conclusions are drawn in Sec. 7..

\section{Broadband Steering Vectors}

An $M$-element array of omnidirectional sensors located at positions $\mathbf{r}_{m}, m=1 \ldots M$ collects a signal vector $\mathbf{x}(t) \in \mathbb{C}^{M}$, with the continuous time variable $t$. If a far field source illuminates the array such that the signal at the origin $\mathbf{r}=\underline{0}$ is $s(t)$ and we neglect attenuation, then

$$
\mathbf{x}(t)=\left[\begin{array}{c}
s\left(t-T_{1}\right) \\
s\left(t-T_{2}\right) \\
\vdots \\
s\left(t-T_{M}\right)
\end{array}\right]=\left[\begin{array}{c}
\delta\left(t-T_{1}\right) \\
\delta\left(t-T_{2}\right) \\
\vdots \\
\delta\left(t-T_{M}\right)
\end{array}\right] * s(t)
$$

with $*$ denoting convolution and delays $T_{m}=\frac{1}{c} \mathbf{k}^{\mathrm{T}} \mathbf{r}_{m}$, $m=1 \ldots M$, where $\mathbf{k}$ is the normal vector of the source's wave front, and $\mathbf{k} / c$ is known as the slowness vector of the source.

Sampling $\mathbf{x}(t)$ with a sampling period $T_{s}$ yields $\mathbf{x}[n]$, with discrete time index $n$ such that $t=n T_{s}$. The assumption is a per- 
fectly bandlimited signal $s(t)$, such that the interpolation function underlying the sampling process is a sinc. With

$$
\mathbf{x}[n]=\left[\begin{array}{c}
\delta\left[n-\tau_{1}\right] \\
\delta\left[n-\tau_{2}\right] \\
\vdots \\
\delta\left[n-\tau_{M}\right]
\end{array}\right] * s[n]=\mathbf{a}[n] * s[n]
$$

and normalised delays $\tau_{m}=T_{m} / T_{s}$, the ideal fractional delays $\delta\left[n-\tau_{m}\right]$,

$$
\delta[n-\tau]=\left\{\begin{array}{lll}
\frac{\sin (\pi(n-\tau))}{\pi(n-\tau)} & , & n \neq \tau \\
1 & , & n=\tau
\end{array}\right.
$$

are now sinc functions which not necessarily remain sampled in the sinc's zero crossing, and therefore generally possess infinite support. The quantity $\mathbf{a}[n]$ in (2) is referred to as broadband steering, and consists of a number of different fractional delays of the type in (3).

A signal model for a scenario with $L$ independent far field broadband sources $s_{l}[n], l=1 \ldots L$, each characterised by a broadband steering vector $\mathbf{a}_{l}[n]$, therefore becomes

$$
\mathbf{x}[n]=\sum_{l=1}^{L} \sum_{v=0}^{\infty} \mathbf{a}_{l}[v] s_{l}[n-v]+\mathbf{v}[n],
$$

with $\mathbf{v}[n]$ representing spatially and temporally uncorrelated noise with covariance $\mathscr{E}\left\{\mathbf{v}[n] \mathbf{v}^{\mathrm{H}}[n]\right\}=\sigma_{v}^{2} \mathbf{I}$. To capture information contained in the data vector $\mathbf{x}[n]$ requires a space-time covariance matrix $\mathbf{R}[v]=\mathscr{E}\left\{\mathbf{x}[n] \mathbf{x}^{\mathrm{H}}[n-v]\right\}$ with lag parameter $v$. Its Fourier pair, the cross-spectral density matrix $\mathbf{R}(z)=$ $\sum_{v} \mathbf{R}[v] z^{-v}$ or short $\mathbf{R}[v] \circ \longrightarrow \mathbf{R}(z)$,

$$
\mathbf{R}(z)=\sum_{l=1}^{L} \mathbf{a}(z) \mathbf{a}^{\mathrm{H}}\left(z^{-1}\right) R_{l}(z)+\sigma_{v}^{2} \mathbf{I}
$$

with $R_{l}(z)$ the power spectral density of the $l$ th source, forms a polynomial matrix.

Some broadband array methods directly utilise the broadband steering vector. In e.g. [3], broadband steering vectors are used to presteer array data. The parametric covariance matrix approach in $[2,10]$ presteers the data prior to scanning for maximised eigenvalues in the resulting covariance matrix. For the polynomial MUSIC algorithm in [1], a polynomial eigenvalue decomposition [11] of the space-time covariance matrix in (5) identifies the noise-only subspace, which can then be scanned using broadband steering vectors, in analogy to the narrowband MUSIC algorithm [12]. Thus, the accuracy of the broadband steering vector implementation impacts crucially on all of these applications.

\section{Fractional Delay Filters}

Based on the idea fractional delay and an error metric defined in Sec. 3.1, this section reviews various implementation methods for fractional delay filters, including windowed sinc functions in Sec. 3.2 and the Farrow structure [8] in Sec. 3.3.

\subsection{Ideal Delay and Performance Metric}

With ideal fractional delay defined by (3),

$$
f_{\text {ideal }}[n]=\delta[n-\tau] \quad \text {, }
$$

and the Fourier pair $\delta[n] \circ-\bullet 1$, the Fourier transform of the fractional delay yields

$$
F_{\text {ideal }}\left(e^{j \Omega}\right)=1 \cdot e^{-j \Omega \tau}
$$

with a group delay $\gamma_{\text {ideal }}=\tau$. Based on this ideal delay, an error metric for an arbitrary fractional delay filter approximation $f[n]$ can be defined as

$$
S_{e e}\left(e^{j \Omega}\right)=\left|F_{\text {ideal }}\left(e^{j \Omega}\right)-F\left(e^{j \Omega}\right)\right|^{2}
$$

with $F\left(e^{j \Omega}\right) \bullet-\circ f[n]$, such that $S_{e e}\left(e^{j \Omega}\right)$ is a quadratic error type metric for the approximation of $f_{\text {ideal }}[n]$ by $f[n]$.

\subsection{Windowed Sinc Methods}

With the ideal fractional delay in (6) possessing infinite support, in general a window $w_{N}[n]$ and time delay is applied to create a causal filter of length $2 N$,

$$
f[n]=f_{\text {ideal }}[n-\tau-N] w_{N}[n-\tau-N]
$$

In the simplest case, a rectangular window $w_{N}[n]=p_{N}[n]$ performs a truncation according to

$$
p_{N}[n]=\left\{\begin{array}{lll}
1 & , & |n| \leq N \\
0 & , & |n|>N
\end{array} .\right.
$$

The resulting discrete prolate spheroidal sequence $f[n]$ provides an approximation of $f_{\text {ideal }}[n]$ that generally improves with $N$ at lower frequencies. However, independent from $N$, the performance degrades due Gibbs phenomena as the Nyquist frequency is approached [13].

To enhance the approximation of an ideal fractional delay, a tapered window can be introduced [4,7], using, for example, a Hann window

$$
w_{N, \operatorname{Hann}}[n]=\cos ^{2}\left(\frac{\pi n}{2 N}\right) p_{N}[n] \quad .
$$

By using such windowing techniques, the ripple in the frequency response can be reduced, lowering the error metric in (8) at lower frequencies.

\subsection{Farrow Structure}

The idea of the Farrow structure [8] is based on a polynomial approximation approach between input samples. Consisting of $M+1$ sections of $L$ th-order FIR filters $C_{m}(z), m=0 \ldots M$, which provide an interpolation between input samples, Fig. 1 shows the diagram of the Farrow structure. The fractional delay 


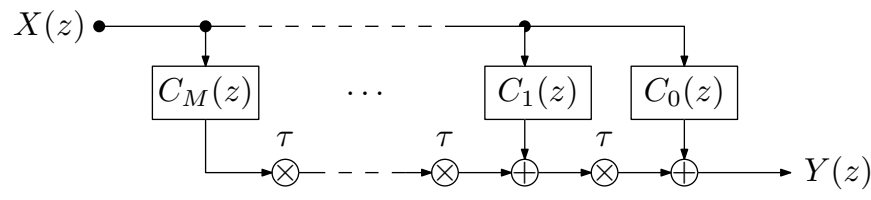

Figure 1. Farrow structure with $M+1$ subsystems of order $L$ approximating a fractional delay $\tau$ between input $X(z)$ and output $Y(z)$.

of the structure is given by the single fractional delay parameter $\tau$, leading to a transfer function

$$
F(z)=\sum_{m=0}^{M} C_{m}(z) \tau^{m}
$$

For a given fractional delay $\tau, F(z)$ is fixed. The magnitude response of the Farrow structure is flat at low frequencies only, thus limiting its applicability to broadband problems that extend beyond lowpass-type signals.

\section{Filter Bank Approach}

Exploiting the high accuracy of various fractional delay filters reviewed in Sec. 3. for lowpass signals, the filter bank based structure in Fig. 2 is proposed as an implementation framework for fractional delay filters. In this structure, the input signal is split into $K$ different frequency bands using an analysis filter bank with filters $H_{k}(z), k=1 \ldots K$. Undecimated, the subband signals are frequency shifted by $\Omega_{k}$ such that the fractional delay filters are applied to lowpass signals in every branch. After fractionally delaying the subband signals, the frequency shifts are reversed, and signal are combined using a suitable bank of synthesis filters $G_{k}(z)$.

For memory and computational simplicity, this analysis filter can be derived from a common lowpass prototype filter by means of a modulating transform. We here use generalised discrete Fourier transform (GDFT) modulated filter banks, which offer advantages over other modulations in terms of subband uniformity and the ability to implement a near-perfect paraunitary system, where the synthesis filters $G_{k}(z)$ can be derived by time reversal from the analysis filters [14]. The prototype filter can be designed using a least-squares approach [14], whereby the reconstruction error of the filter bank is a design criterion that is optimised. Therefore, depending on the quality of the prototype filter, and therefore its length and complexity, different levels of reconstruction errors can be achieved for the filter bank.

The characteristic of a sample filter bank with $K=16$ subbands is shown in Fig. 3. The bandpass nature of the subband signals motivates the modulation by

$$
\Omega_{k}=\frac{(2 k-1) \pi}{K} \quad k \in \mathbb{Z}, k=1 \cdots K,
$$

which translates every subband in frequency to sit symmetrically around $\Omega=0$.

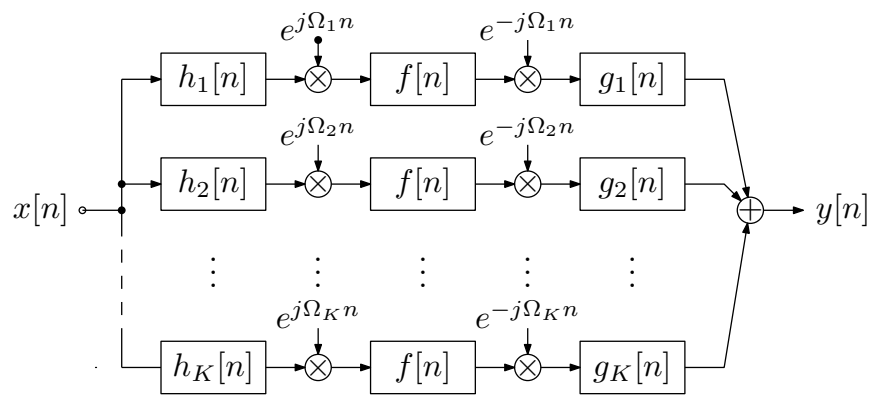

Figure 2. Proposed subband-based fractional delay filter with analysis filter bank stage with analysis filters $h_{k}[n] \circ-\bullet H_{k}(z)$, a modulation stage, the fractional delay filters $f[n]$, a demodulation stage, followed by a synthesis filter bank with synthesis filters $g_{k}[n] \circ \longrightarrow \bullet G_{k}(z)$.

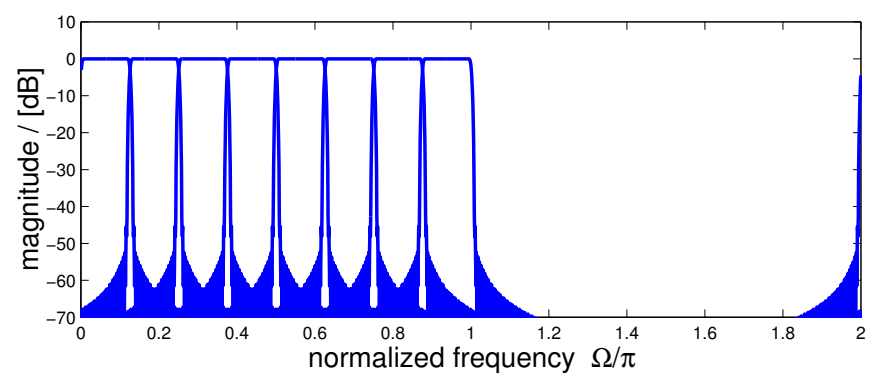

Figure 3. Characteristic of the lower $K / 2$ analysis filters $H_{k}\left(e^{j \Omega}\right) \bullet-\circ h_{k}[n]$ of a $K$ channel filter bank.

\section{Complexity Considerations}

Before assessing and comparing the accuracy of various fractional delay filter methods discuss in Sec. 3., this section will analyse the computational complexity of these approaches.

The straightforward windowed sinc function requires

$$
C_{\text {window }}=2 N
$$

multiply accumulates (MACs), which is independent of the particular window function such as Hann or a rectangular window, as the coefficients can be saves readily multiplied onto sinc values. The Farrow structure with its polynomial order $M$ and filter length $L$ consumes

$$
C_{\text {Farrow }}=L(M+1)+M
$$

MACs per sampling period, which can be substantial if $L \approx N$.

Finally, the computational complexity for the filter bank approach in its most efficient implementation based on a modulated filter bank in polyphase implementation [15], which requires

$$
C_{F B}=2 L_{\mathrm{p}}+4 K \log _{2} K,
$$

per filter bank and sampling period. In (16), $L_{\mathrm{p}}$ is the order of filter bank's prototype filter and $K$ is the number of subbands. For the proposed approach, 2 filter bank operations and a total of $K$ fractional delay filter implementations according to Fig. 2 therefore lead to

$$
C_{\text {proposed }}=2 C_{\mathrm{FB}}+K \cdot C,
$$




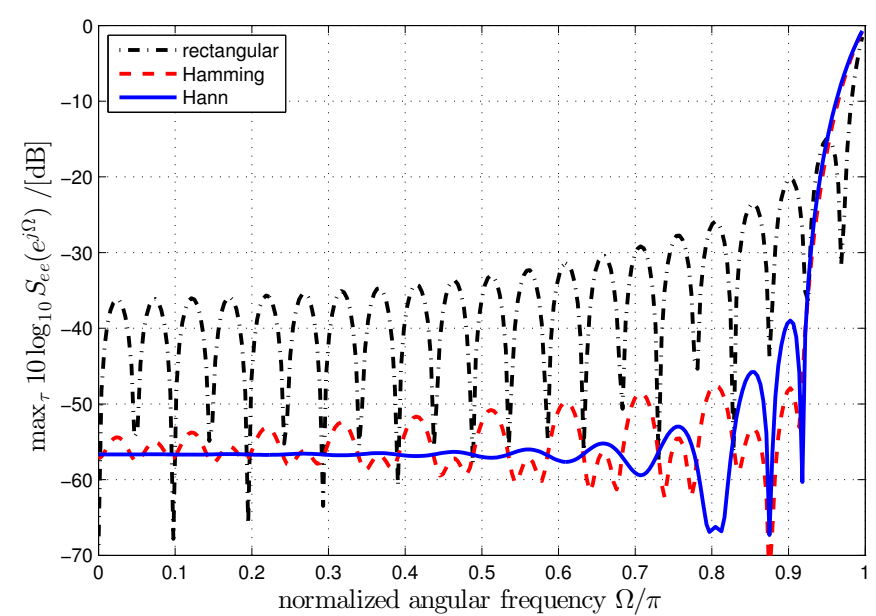

Figure 4. Approximation error $S_{e e}\left(e^{j \Omega}\right)$ maximised over fractional delay $\tau$, and dependent on normalised angular frequency $\Omega$.

whereby $C$ takes on the value in (14) or (15), depending on which fractional delay scheme is integrated into the proposed architecture.

Considering (14), (15) and (17), it is clear that the filter bank based approach is significantly less expensive compared to a direct implementation. Therefore, the performance analysis in Sec. 6. will have to demonstrate if the computational cost that needs to be afforded for the proposed approach is worthwhile.

\section{Simulations and Numerical Results}

For the windowed approaches, Fig. 4 provides some preliminary results on the maximisation of the error $S_{e e}\left(e^{j \Omega}\right)$ over the fractional delay as a variable. The rectangular window performes worst, and improvements can be made at no arising cost through the use of tapered windows. As an alternative to the Hann window, we also show a Hamming window, which, however performs slightly worse than its competitor.

The approximation error for a truncated sinc function with $N=100$ is shown in Fig. 5, where a maximum error is reached for a fractional delay of $\tau=\frac{1}{2}$ and frequencies approaching the Nyquist rate. The degradation towards the Nyquist rate is shared by the Hann-windowed sinc function in Fig. 6, also with window $N=100$, and a Farrow structure with polynomial order $M=3$ in Fig. 7. While the Farrow structure for a low polynomial degree does not perform well for higher frequencies, it significantly exceeds both the rectangular and Hannwindowed sinc approach, whereby the Hann-window offers advantages over the rectangular window at almost no cost.

To quantitatively assess the performance of the above fractional delay filters, we defined two approximation errors. A first error represents the average deviation from an ideal delay over the entire band,

$$
\sigma_{\text {full }}^{2}=\frac{1}{\pi} \int_{0}^{\pi} S_{e e}\left(e^{j \Omega}\right) d \Omega
$$

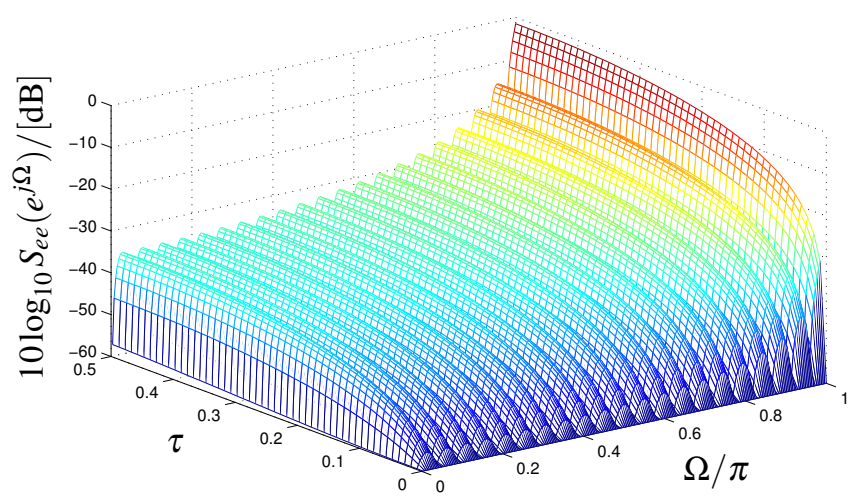

Figure 5. Approximation error for truncated sinc function with $N=100$.

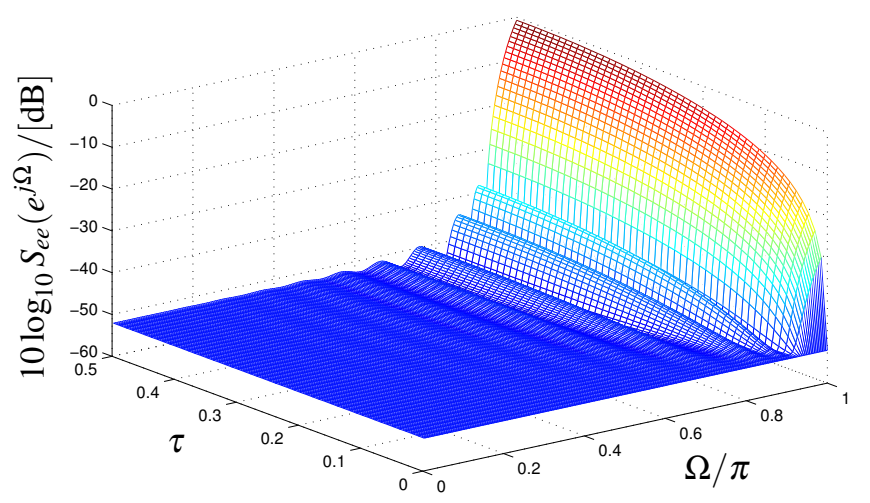

Figure 6. Approximation error for Hann windowed sinc function with $N=100$.

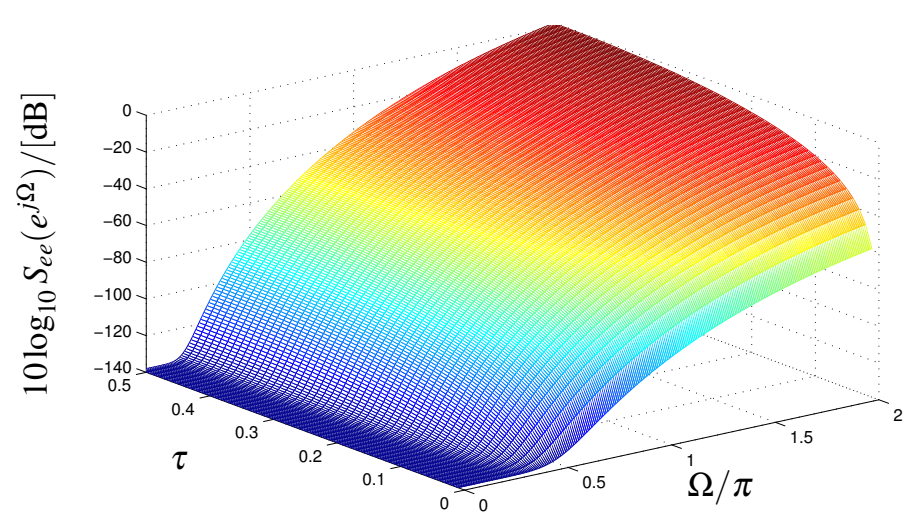

Figure 7. Approximation error for Farrow structure for $M=3$.

while a second error removes the highest octave — where according to Figs. 5-7 the approximation is least accurate to calculate

$$
\sigma_{\text {half }}^{2}=\frac{2}{\pi} \int_{0}^{\pi / 2} S_{e e}\left(e^{j \Omega}\right) d \Omega
$$

over the lower half of the spectrum only. The resulting measure for the windowed sinc function and the Farrow structure are shown in Fig. 8. This provides a clear indication that a 


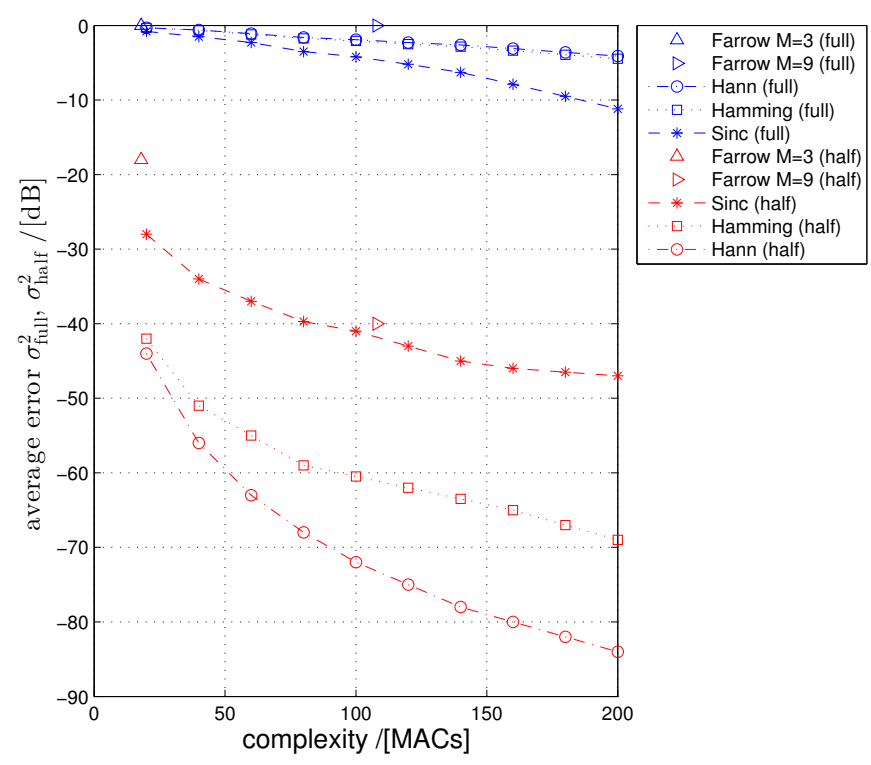

Figure 8. Error performance versus complexity for various fractional delay filter approaches.

restriction to lowpass signals provides accurate results, particularly for the Hann window, while state-of-the-art methods are unsuitable when applied over the entire Nyquist band.

For the proposed filter bank approach to a fractional delay implementation, Fig. 9 shows the combination of a $K=16$ channel filter bank with an $M=3$ order Farrow structure to implement the fractional delay in the frequency-shifted subbands. As the results in Fig. 9, the error is uniformly low with a maximum error $S_{e e}\left(e^{j \Omega}\right)$ of $-55 \mathrm{~dB}$ across all frequencies $\Omega$ and fractional delays $\tau$. Here, $S_{e e}\left(e^{j \Omega}\right)$ consists of two contributions - (i) an error due to inaccuracies on the Farrow structure, and (ii) a reconstruction error within the filter bank. Here, with a reconstruction error of $-55 \mathrm{~dB}$ [14], the latter dominates. This is underlined by the same error of $-55 \mathrm{~dB}$ that is obtained in combination with a Hann windowed sinc function, and a Farrow structure of order $M=9$. In contrast, embedding the sinc function characterised in Fig. 5 into the subbands yields an approximation error of approximately $-37 \mathrm{~dB}$; i.e. for this case, the fractional delay filter is sufficiently crude to dominate the overall error of the system. This is also supported by the approximation error $\sigma_{\text {full }}^{2}$ in (18), which is summarised for the various fractional delay filter methods in Tab. 1. The fractional delay filter methods using the rectangularly truncated sinc is given a length of equivalent complexity to the displayed filter bank based methods.

Since the undecimated filter bank approach is costly in terms of computations, the filter bank can design such that it is just sufficiently good to match the desired approximation error for the fractional delay filter $f[n]$. This ensures that the system is not over-designed, and that the cost of the filter bank can be kept as low as possible.

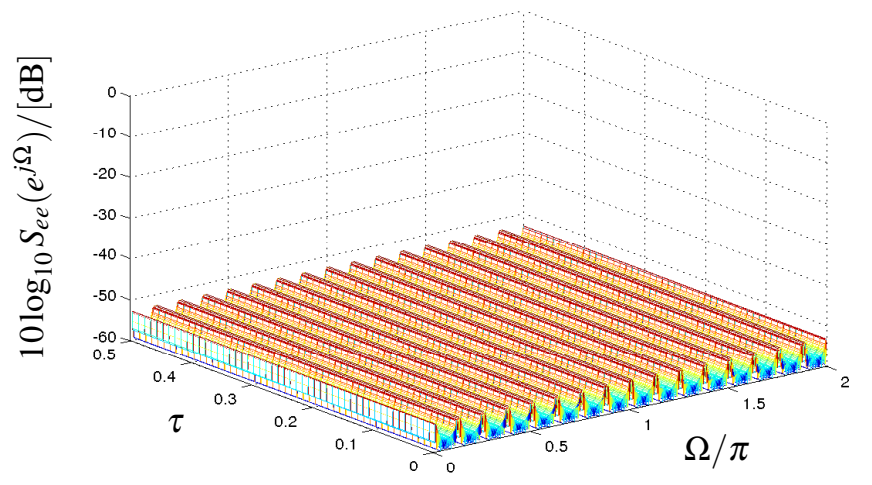

Figure 9. Approximation error for filter bank approach with with $K=16$, and an $L=3$ order Farrow filter as subband fractional delay $f[n]$.

\begin{tabular}{|l||r|}
\hline method & $\sigma_{\text {full }}^{2} /[\mathrm{dB}]$ \\
\hline \hline sinc & -21.1 \\
Farrow, $L=9$ & -0.5 \\
fiba \& sinc & -38.0 \\
fiba \& Hann & -55.3 \\
fiba \& Farrow $M=3$ & -55.5 \\
fiba \& Farrow $M=9$ & -55.8 \\
\hline
\end{tabular}

Table 1. Table of average errors over entire Nyquist band for different fractional delay filter implementations. The filter band (fiba) methods use a $K=16$ channel filter bank with a reconstruction error of approx $-56 \mathrm{~dB}$. .

\section{Conclusions}

The need for accurate broadband steering vectors for applications such as broadband angle of arrival estimation has motivated the implementation of fractional delay filters that can approach the ideal fractional delay over a large bandwidth. Since state-of-the-art fractional delays such as windowed sinc and Farrow filters perform best at low frequencies, we have combined these filters with a modified filter bank, whereby undecimated subband signals are modulated such that only a small lowpass region is active in each subband. These can then be accurately delayed by any of the established methods.

Simulations indicate that accuracy can be achieved across the entire bandwidth, and that the approximation error w.r.t. an ideal delay is either limited by the fractional delay filter or the reconstruction error of the filter bank. The final paper will provide additional details on the complexity, and the trade-off and optimal selection of the filter bank.

\section{References}

[1] M. Alrmah, S. Weiss, and S. Lambotharan, "An extension of the music algorithm to broadband scenarios using polynomial eigenvalue decomposition," in 19th European Signal Processing Conference, Barcelona, Spain, August 2011, pp. 629-633.

[2] J. Dmochowski, J. Benesty, and S. Affes, "Direction of arrival estimation using the parameterized spatial correlation matrix," IEEE Transactions on Audio, Speech, and Language Processing, vol. 15, no. 4, pp. 1327-1339, May 2007.

[3] P. Murphy, A. Krukowski, and A. Tarczynski, "An efficient fractional sample delayer for digital beam steering," in IEEE International Conference on Acoustics, Speech, and Signal Processing, vol. 3, Munich, Germany, April 1997, pp. 2245-2248. 
[4] A. Yardim, G. Cain, and P. Henry, "Optimal two-term offset windowing for fractional delay," Electronics Letters, vol. 32, no. 6, pp. 526-527, March 1996.

[5] T. I. Laakso, V. Välimäki, M. Karjalainen, and U. K. Laine, "Splitting the Unit Delay," IEEE Signal Processing Magazine, vol. 13, no. 1, pp. 30-60, January 1996.

[6] A. Papoulis, Signal Analysis. New York: McGraw-Hill, 1984.

[7] J. Selva, "An efficient structure for the design of variable fractional delay filters based on the windowing method," IEEE Transactions on Signal Processing, vol. 56, no. 8, pp. 37703775, August 2008.

[8] C. W. Farrow, "A continuously variable digital delay element," in IEEE International Symposium on Circuits and Systems, vol. 3, Espoo, FInland, June 1988, pp. 2641-2645.

[9] S. Weiss, S. R. Dooley, R. W. Stewart, and A. K. Nandi, "Adaptive Equalisation in Oversampled Subbands," IEE Electronics Letters, vol. 34, no. 15, pp. 1452-1453, July 1998.

[10] M. Souden, J. Benesty, and S. Affes, "Broadband source localization from an eigenanalysis perspective," IEEE Transactions on Audio, Speech, and Language Processing, vol. 18, no. 6, pp. 1575-1587, August 2010.

[11] J. G. McWhirter, P. D. Baxter, T. Cooper, S. Redif, and J. Foster, "An EVD Algorithm for Para-Hermitian Polynomial Matrices," IEEE Transactions on Signal Processing, vol. 55, no. 5, pp. 2158-2169, May 2007.

[12] R. O. Schmidt, "Multiple emitter location and signal parameter estimation," IEEE Transactions on Antennas and Propagation, vol. 34, no. 3, pp. 276-280, March 1986.

[13] M. Sac and M. Blok, "Gain deficit effect in the fractional delay filter design by the window method," in Proc. SPIE Photonics Applications in Astronomy, Communications, Industry, and High-Energy Physics Experiments, vol. 75021G, August 2009, pp. 1-6.

[14] M. Harteneck, S. Weiss, and R. Stewart, "Design of near perfect reconstruction oversampled filter banks for subband adaptive filters," IEEE Transactions on Circuits and Systems II: Analog and Digital Signal Processing, vol. 46, no. 8, pp. 1081-1085, August 1999.

[15] S. Weiss and R. Stewart, "Fast Implementation of Oversampled Modulated Filter Banks," Electronics Letters, vol. 36, no. 17, pp. 1502-1503, August 2000. 